\title{
A self-similar solution of expanding cylindrical flux ropes for any polytropic index value
}

\author{
Hironori Shimazu $^{1 *}$ and Marek Vandas ${ }^{2}$ \\ ${ }^{1}$ Applied Research and Standards Division, Communications Research Laboratory, Koganei, Tokyo 184-8795, Japan \\ ${ }^{2}$ Astronomical Institute, Academy of Sciences, Boční II 1401, 14131 Praha 4, Czech Republic
}

(Received December 6, 2001; Revised May 20, 2002; Accepted May 27, 2002)

\begin{abstract}
We found a new class of solutions for MHD equations that satisfies the condition that cylindrical flux ropes can expand self-similarly even when the polytropic index $\gamma$ is larger than 1 . We achieved this by including the effects of elongation along the symmetry axis as well as radial expansion and assuming that the radial expansion rate is the same as the elongation rate. In previous studies (Osherovich et al., 1993a, 1995), a class of self-similar solutions was described for which cylindrical flux ropes expand only in the medium where $\gamma$ is less than 1 . We compare the models including elongation and excluding elongation observationally by using the WIND key parameters. The difference in the fitting results of the magnetic field between these two models is slight. However the fitting of the velocity is improved when elongation is included and when new geometric parameters that are necessary to represent the elongation are introduced. The values of these parameters are almost the same scale as the radius of flux ropes, which is consistent with the assumption of the isotropic expansion. This new exact solution to a time-dependent two-dimensional MHD problem can also be used to test numerical codes.
\end{abstract}

\section{Introduction}

Interplanetary magnetic flux ropes are structures in which magnetic field vectors rotate in one direction through a large angle. The sun often ejects flux ropes as coronal mass ejections (e.g., Marubashi, 1997). When a flux rope reaches the earth, it often causes a geomagnetic storm (e.g., Tsurutani $e t$ al., 1988). Observationally, Burlaga et al. (1981) first identified them as "magnetic clouds" in interplanetary magnetic field data. Goldstein (1983) proposed explaining the rotation of the interplanetary magnetic field vectors by using a cylindrical force-free flux rope model, and Marubashi (1986) confirmed this by using data taken in interplanetary space. Several methods for determining the geometric and physical parameters of flux ropes, such as the radius and the magnetic field, have been developed. Burlaga (1988) proposed a constant-alpha force-free model for the internal magnetic field configuration of flux ropes. Lepping et al. (1990) developed a nonlinear least squares method to fit observed magnetic field variations to this model.

While the flux rope propagates in interplanetary space, it expands because the ambient pressure decreases with its distance from the sun. The technique of converting time derivatives to self-similarity expansion parameters in MHD (magnetohydrodynamics) equations was originally applied by Bernstein and Kulsrud (1965) and Kulsrud et al. (1965) to the explosion of a supernova. The self-similar approach

*Now at Department of Earth and Space Sciences, Box 351310, University of Washington, Seattle, WA 98195-1310, U.S.A.

Copy right(c) The Society of Geomagnetism and Earth, Planetary and Space Sciences (SGEPSS); The Seismological Society of Japan; The Volcanological Society of Japan; The Geodetic Society of Japan; The Japanese Society for Planetary Sciences. simplifies time-dependent problems and makes them analytically tractable. Low (1982) found a class of self-similar solutions of the expanding solar corona in the spherical coordinates when the polytropic index $\gamma$ is exactly $4 / 3$. Gibson and Low (1998) used the same approach, and presented a theoretical MHD model describing the time-dependent expulsion of a three-dimensional coronal mass ejection.

Osherovich et al. (1993a, 1995) analyzed MHD equations and found a class of self-similar solutions. The solution showed that cylindrical flux ropes expand self-similarly only when $\gamma$ is less than 1 for this class of self-similar solutions. Farrugia et al. (1993) applied this formulation to real interplanetary flux ropes. Osherovich et al. (1993b) interpreted observations of solar wind electrons in interplanetary magnetic flux ropes as having $\gamma$ less than 1 , and that is why flux ropes expand in interplanetary space.

However, coronal mass ejections, or flux ropes, expand in MHD simulations even when $\gamma$ is larger than 1 (Vandas $e t$ al., 1995; Vandas et al., 1996; Wu et al., 1997; Odstrčil and Pizzo, 1999; Vandas and Odstrčil, 2000). Moreover, theoretical models of Chen and Garren (1993) and Chen (1996) showed expanding flux ropes with $\gamma>1$. Recent comprehensive analysis by Gosling (1999) has concluded that an observed negative correlation between the temperature and the density of electrons, on which the derivation of $\gamma$ was based, cannot be a measure of $\gamma$. Skoug et al. (2000a, b) showed that single-point measurements cannot be used to determine $\gamma$ value. To determine $\gamma$ value observationally continues to be a matter for debate (Osherovich, 2001; Gosling et al., 2001).

This paper describes a new class of solutions for MHD equations that satisfies the condition that cylindrical flux 
ropes can expand self-similarly even when $\gamma$ is larger than 1. We achieved this by including effects of elongation along the symmetry axis, which is not included in the previous solutions. This new class of solutions puts new light to the very discussed problem on the relation between $\gamma$ and the expansion. Mainly it emphasizes a role of flux rope elongation for its expansion. We also compare the models including elongation and excluding elongation to interplanetary observations by using the WIND key parameters.

\section{Solution}

The MHD equations are solved as follows:

$$
\begin{gathered}
\frac{\partial \rho}{\partial t}+\nabla \cdot(\rho \boldsymbol{v})=0, \\
\rho \frac{\partial \boldsymbol{v}}{\partial t}+\rho(\boldsymbol{v} \cdot \nabla) \boldsymbol{v}=-\nabla P+\frac{1}{\mu}(\nabla \times \boldsymbol{B}) \times \boldsymbol{B}, \\
\frac{\partial\left(P \rho^{-\gamma}\right)}{\partial t}+(\boldsymbol{v} \cdot \nabla)\left(P \rho^{-\gamma}\right)=0,
\end{gathered}
$$

and

$$
\frac{\partial \boldsymbol{B}}{\partial t}=\nabla \times(\boldsymbol{v} \times \boldsymbol{B}),
$$

where $t$ is time, $\rho$ is mass density, $P$ is pressure, $\mu$ is permeability, $\boldsymbol{v}$ is velocity, and $\boldsymbol{B}$ is the magnetic field. These MHD equations will be solved in cylindrical coordinates $(r$, $\theta, z)$ moving with a flux rope. The cylindrical flux rope has its axis along $z$ and an axial symmetry is assumed, that is, the flux rope has a circular cross section and the quantities do not depend on $\theta$.

\subsection{Previous self-similar solution (without elongation)}

Before showing the new solution, we will review the previous solution. This solution assumes self-similarity and no dependence on $z$ (one-dimension and $r$-dependence only). Following the procedure described in Osherovich et al. (1995), the solutions for Eqs. (1), (3), and (4) were

$$
\begin{gathered}
v_{r}=\eta \dot{y}, \\
B_{\theta}=\left(-\eta f^{\prime} / 2\right)^{1 / 2} y^{-1}, \\
B_{z}=(2 \mu S D)^{1 / 2} y^{-2}, \\
\rho=-D^{\prime} \eta^{-1} y^{-2},
\end{gathered}
$$

and

$$
P=K D y^{-2 \gamma}
$$

where

$$
D=\frac{f+\eta f^{\prime} / 2}{2 \mu S \chi},
$$

and $\chi, S$, and $K$ are positive constants. The center dot means the derivative by the time $t$, and the prime is the derivative by the self-similar parameter $\eta$ satisfying

$$
\eta=r y^{-1},
$$

where $y$ is the evolution function of time. The $f$ is the generating function of $\eta$, satisfying

$$
\begin{gathered}
f^{\prime} \leq 0, \\
f+\eta f^{\prime} / 2 \geq 0,
\end{gathered}
$$

and

$$
\left(f+\eta f^{\prime} / 2\right)^{\prime} \leq 0
$$

From the $r$ component of the equation of motion (2), $y$ satisfies

$$
\begin{gathered}
\ddot{y}=-\frac{d U}{d y}, \\
U= \begin{cases}\frac{K}{2 \gamma-2} y^{2-2 \gamma}+(1 / 2) S y^{-2}+\chi S \ln y & (\gamma \neq 1) \\
(\chi S-K) \ln y+(1 / 2) S y^{-2} & (\gamma=1) .\end{cases}
\end{gathered}
$$

Equation (15) can be regarded as an equation of motion under potential $U$. When $\gamma$ is larger than 1, $U$ takes a minimum value. Thus, flux ropes do not expand but oscillate when $\gamma$ is larger than 1 .

\subsection{New solution (with elongation)}

In this study we include effects of axial elongation $(z-$ dependence) as well as radial expansion. The $v_{\theta}$ and $B_{r}$ are assumed to be 0 . An additional self-similar parameter $\xi$ is introduced by

$$
\xi=z y^{-1} .
$$

This is consistent with the assumption that the radial expansion rate is the same as the elongation rate. We will solve Eqs. (1)-(4) that satisfy

$$
\frac{\partial f}{\partial \xi}=0
$$

for the simplest case. A new solution for Eqs. (1), (3), and (4) is given by

$$
\begin{gathered}
v_{r}=\eta \dot{y}, \\
v_{z}=\xi \dot{y}, \\
B_{\theta}=\left(-\eta f^{\prime} / 2\right)^{1 / 2} y^{-2}, \\
B_{z}=(2 \mu S D)^{1 / 2} y^{-2}, \\
\rho=-G^{\prime} \eta^{-1} y^{-3},
\end{gathered}
$$

and

$$
P=K G y^{-3 \gamma}
$$

where $G$ is a function of $\eta$ and $\xi$.

From the $r$ component of Eq. (2), $U$ is expressed as

$$
U= \begin{cases}\frac{K}{3 \gamma-3} y^{3-3 \gamma}+\left(D^{\prime} / G^{\prime}\right) S(1-\chi) y^{-1} & (\gamma \neq 1) \\ -K \ln y+\left(D^{\prime} / G^{\prime}\right) S(1-\chi) y^{-1} & (\gamma=1)\end{cases}
$$

where $D$ is also defined by Eq. (10) in this new solution. The $\theta$ component of Eq. (2) is zero under the condition (18). From the $z$ component of Eq. (2), we get

$$
U= \begin{cases}\frac{K}{3 \gamma-3} y^{3-3 \gamma} \frac{\eta(\partial G / \partial \xi)}{\xi(\partial G / \partial \eta)} & (\gamma \neq 1) \\ -K \ln y \frac{\eta(\partial G / \partial \xi)}{\xi(\partial G / \partial \eta)} & (\gamma=1) .\end{cases}
$$




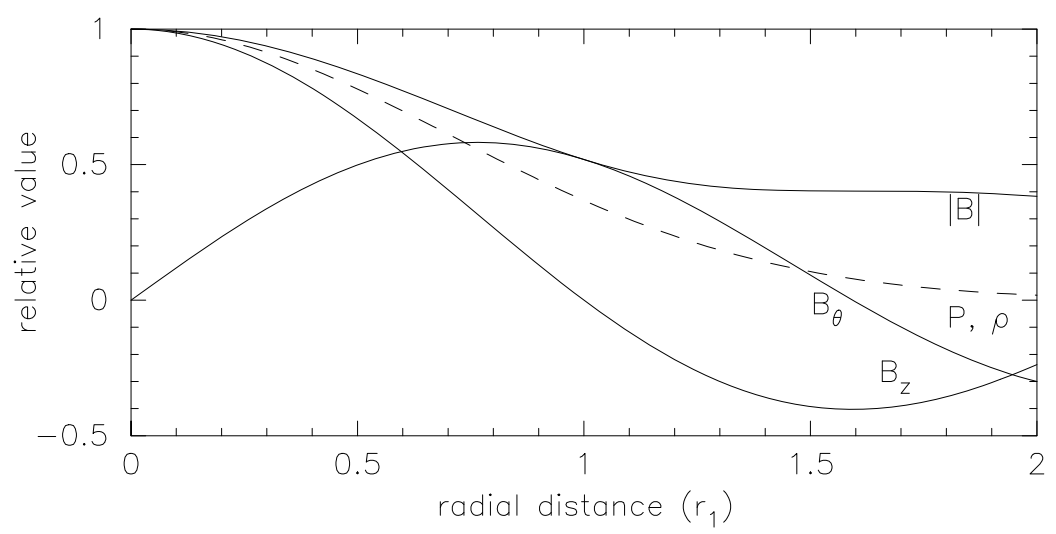

Fig. 1. Profiles of quantities in the $r$ direction on the $z=0$ plane obtained at an arbitrary time. The radial distance is measured in the flux rope radius $\left(r_{1}\right)$. It is 0.0 at the flux rope axis and 1.0 at the boundary.

For Eq. (25) to agree with Eq. (26), the conditions

$$
\chi=1
$$

and

$$
\frac{1}{\eta} \frac{\partial G}{\partial \eta}=\frac{1}{\xi} \frac{\partial G}{\partial \xi}
$$

must be satisfied. From Eq. (28) we get

$$
G=a \exp \left[-c\left(\eta^{2}+\xi^{2}\right)\right],
$$

where $a$ and $c$ are constants. Thus, the pressure distribution is expressed as

$$
P=K a \exp \left[-c\left(r^{2}+z^{2}\right) / y^{2}\right] y^{-3 \gamma} .
$$

The $\rho$ is given from Eq. (23) by

$$
\rho=2 a c \exp \left[-c\left(r^{2}+z^{2}\right) / y^{2}\right] y^{-3} .
$$

For the density to be positive anywhere and to be zero in infinitely distant regions, $a$ and $c$ must both be positive. In this new solution we do not need the condition (14), under which the density was positive or zero in the previous solution. The temperature $T$ is expressed as

$$
T=\frac{m K}{2 k c} y^{-3 \gamma+3},
$$

where $k$ is Boltzmann's constant and $m$ is the average mass of the solar wind particles.

There is also another solution for Eq. (28):

$$
G=-a\left(\eta^{2}+\xi^{2}\right)+c,
$$

which gives

$$
T=\frac{m K}{2 k a}\left[-a\left(\eta^{2}+\xi^{2}\right)+c\right] y^{-3 \gamma+3} .
$$

This solution is not realistic because in some cases $T$ is negative.

Finally, we get

$$
U= \begin{cases}\frac{K}{3 \gamma-3} y^{3-3 \gamma} & (\gamma \neq 1) \\ -K \ln y & (\gamma=1) .\end{cases}
$$

This expression shows that $U$ is a monotonically decreasing function of $y$. Thus, flux ropes obeying this solution expand for any $\gamma$ value.

Equation (15) with the given potential (35) can be integrated yielding

$$
d y / d t= \begin{cases} \pm\left[\frac{2 K}{3-3 \gamma} y^{3-3 \gamma}+c_{1}\right]^{1 / 2} & (\gamma \neq 1) \\ \pm\left[2 K \ln y+c_{1}\right]^{1 / 2} & (\gamma=1)\end{cases}
$$

where $c_{1}$ is a constant. In case $\gamma=5 / 3$, we can integrate this equation further, and get

$$
y=\left[c_{1}\left(t+t_{0}\right)^{2}+K / c_{1}\right]^{1 / 2},
$$

where $t_{0}$ is a constant; $v_{r}=v_{z}=0$ everywhere at $t=-t_{0}$. We took the plus sign assuming that $y$ is a monotonically increasing function of $t$. In our solution $y^{-1}$ is finite at $t=-t_{0}$, while in the previous solution $y^{-1}$ is infinitely large. Thus, an infinitely large magnetic field strength is suppressed in our solution.

Figure 1 shows sample profiles of quantities in the $r$ direction when we take $f$ as it is most frequently used in a description of a cylindrical flux rope:

$$
f=B_{1}^{2}\left\{J_{0}^{2}\left(\alpha_{1} \eta\right)+J_{1}^{2}\left(\alpha_{1} \eta\right)\right\},
$$

where $J_{0}$ and $J_{1}$ are Bessel functions of the first kind of order 0 and 1 , and $B_{1}$ and $\alpha_{1}$ are constants. One can directly compare this figure with a similar figure shown by Vandas and Odstrčil (2000, figure 7) for the previous solution without elongation, but with the same generating function. While in the previous solution the density and pressure drop to zero at the flux rope's boundary, these quantities remain positive in the new solution.

\subsection{Features of the new solution}

The most important effect of elongation of the axial length appears in the azimuthal component of the magnetic field:

$$
B_{\theta} \sim y^{-1} \quad \text { (excluding elongation) }
$$

is modified into

$$
B_{\theta} \sim y^{-2} \text { (including elongation). }
$$


The difference of $B_{\theta}$ dependence on $y$ comes from the volume increase in the axial direction. This is the essential effect of the elongation. Chen (1996) suggested the lack of a three-dimensional effect as the reason the Osherovich et al. (1995) solution does not expand when $\gamma$ is greater than 1. This statement agrees with our solution.

We consider that a flux rope has a finite volume. The radius $r_{1}=r_{0} y$ and the axial length $l=l_{0} y$ are determined by the initial radius $r_{0}$ and the initial length $l_{0}$ (at $t=-t_{0}$ ), respectively. $r_{1}$ is taken for $B_{z}$ to be zero at the boundary.

Kumar and Rust (1996) constructed a flux rope model for interplanetary magnetic clouds assuming that the total magnetic helicity is conserved. Our model agrees with their model in the dependence on $l$ of the magnetic field strength, mass density, radius, and volume. Their results also showed that a flux rope evolves self-similarly, which agrees with our assumption.

Chen (1996) investigated the dynamics of flux ropes. His model starts with an equilibrium state of pressure between a flux rope and the ambient medium. He treated propagation in interplanetary space by the change of physical parameters of the ambient medium. In our model, we do not assume an initial equilibrium as other self-similar models do not assume. However, our model can trace a flux rope evolution during propagation in interplanetary space by the decreases of the ambient pressure and density with time.

In our solution the magnetic flux is expressed as

$$
\int_{0}^{r_{1}} B_{z} 2 \pi r d r=\int_{0}^{r_{0}} 2 \pi(2 \mu S D)^{1 / 2} \eta d \eta .
$$

The magnetic flux is independent of $y$ and is conserved at all times. The magnetic energy is expressed as

$$
\begin{aligned}
& \frac{1}{2 \mu} \int_{0}^{l} \int_{0}^{r_{1}}\left(B_{\theta}^{2}+B_{z}^{2}\right) 2 \pi r d r d z \\
& \quad=\frac{1}{y} \frac{\pi}{\mu} \int_{0}^{l_{0}} \int_{0}^{r_{0}}\left(2 \mu S D-\eta f^{\prime} / 2\right) \eta d \eta d \xi .
\end{aligned}
$$

The magnetic energy decreases with increasing $y$ and with increasing time. In the models of Kumar and Rust (1996) and Chen (1996), the magnetic energy decreases as the flux rope expands. Our solution agrees with their results.

The $r$ component of the Lorentz force is given by

$$
(1 / \mu)((\nabla \times \boldsymbol{B}) \times \boldsymbol{B})_{r}=(\chi-1) S D^{\prime} y^{-5} .
$$

The other components are 0 . Thus, when $\chi=1$ (Eq. (27)), a force-free state is maintained at all times. Expansion is caused by the pressure gradient force. The ambient and internal pressures control the expansion. This point is different from the Osherovich et al. (1995) solution, in which the force-free state occurred only for one specific time.

Our solution is different from the models of Kumar and Rust (1996) and Chen (1996) in maintaining the force-free state. In their models the force-free state was not maintained. The difference comes from the internal magnetic field configuration, which may be caused by the curvature of the cylindrical axis. If we consider a curved tube as a flux rope like Kumar and Rust (1996) or Chen (1996), it may be difficult to maintain the force-free state.

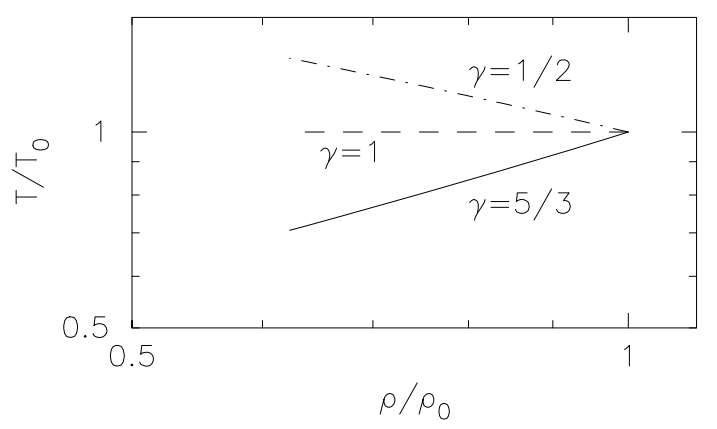

Fig. 2. Relation between the temperature and density while a virtual spacecraft moves across an evolving flux rope for 20 hours. $T_{0}$ and $\rho_{0}$ are the temperature and density observed at the spacecraft encounter, respectively. The parameters of the flux rope geometry are $R_{0}=0.25 \mathrm{AU}$ (radius of the flux rope at spacecraft encounter), $t_{0}=100$ hours (defined in Eq. (37)) $\theta_{1}=45.0$ degrees (ecliptic latitude angle of the symmetry axis), $\phi_{1}=45.0$ degrees (ecliptic longitude angle of the symmetry axis measured from the line connecting the sun and the earth), and $p=0.5$ (impact parameter).

As with some other self-similar solutions, velocity grows without limit as $r$ or $z$ increases. Although we consider finite volume around the origin as a flux rope, infinitely large velocity in infinitely distant regions (for large $r$ as well as for large $|z|$ ) seems strange. However, in our opinion it is significant to show possible expansion in the medium where $\gamma$ is greater than 1 in the same framework of self-similarity as Osherovich et al. (1995) showed.

The relation between $T$ and $\rho$ is considered. From Eq. (32) $T$ decreases with time when $\gamma$ is greater than 1 . Thus $T$ and $\rho$ show a positive correlation in this case. If $\gamma$ is less than 1 , they show a negative correlation. If $\gamma$ is equal to $1, T$ is constant. The relation between the correlation and $\gamma$ is the same as the previous solution.

We examined this fact with a virtual spacecraft passing. Figure 2 shows an example of the relation between $T$ and $\rho$ while a virtual spacecraft moves across an evolving flux rope. In this figure the slope of the curve represents $\gamma$. The results are consistent with the above analytical consideration.

The anti-correlation between the electron temperature and density observed by Osherovich et al. (1993b) cannot be reproduced if $\gamma$ is greater than 1 . The anti-correlation seems possible when the spacecraft moves rapidly to the center of the flux rope, but the correlation is positive when it moves from the center to the outer side. When we put them together, the correlation becomes positive. However in this paper we do not discuss determination of $\gamma$ observationally because it would carry us too far away from the purpose of this paper. We may leave the details to Osherovich (2001) and Gosling et al. (2001). The purpose of this paper is to show that cylindrical flux ropes can expand self-similarly even when $\gamma$ is larger than 1 .

\section{Application to Interplanetary Flux Ropes 3.1 Method}

Applying the new solution, we can construct a new model to be fitted to real interplanetary magnetic field data. In this section we consider which model can fit better to the real observational data, the model excluding elongation or the new model including elongation. 


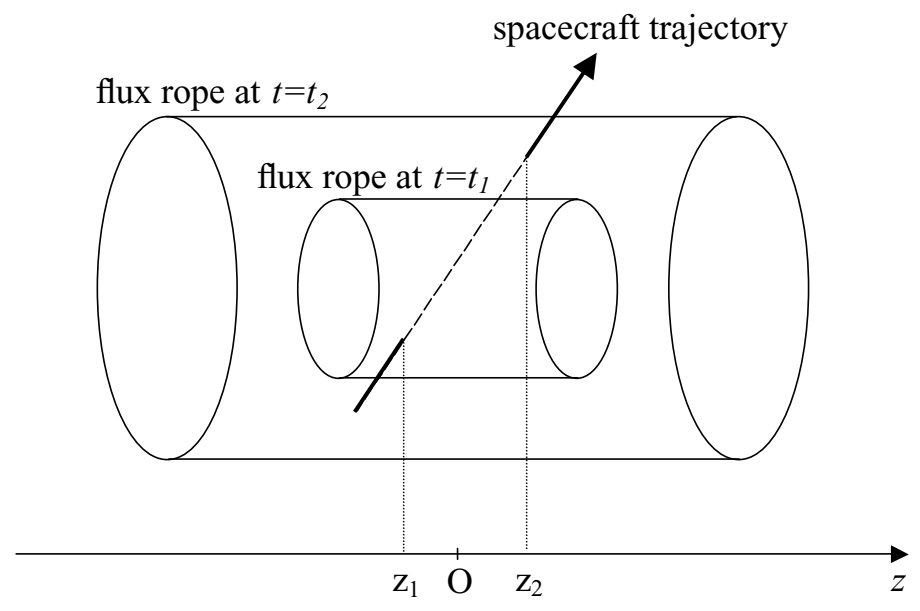

Fig. 3. New parameter $z_{1}$ is the axial distance from the center of the flux rope to the location of the spacecraft encounter and $z_{2}$ is the one from the center to the location where the spacecraft detached from the flux rope.

As already shown, the most important effect of elongation of the axial length appears in the $\theta$ component of the magnetic field. When $f$ is represented by Eq. (38), we substitute it into Eqs. (6) and (7). Then, the magnetic field of the model excluding elongation is expressed as

$$
\begin{aligned}
& B_{\theta}=B_{0} J_{1}\left(\alpha r /\left(1+t / t_{0}\right)\right) /\left(1+t / t_{0}\right), \\
& B_{z}=B_{0} J_{0}\left(\alpha r /\left(1+t / t_{0}\right)\right) /\left(1+t / t_{0}\right)^{2},
\end{aligned}
$$

where $B_{0}$ and $\alpha$ are constants (Farrugia et al., 1992).

The magnetic field of the new model including elongation is expressed by substituting Eq. (38) into Eqs. (21) and (22) as

$$
\begin{aligned}
& B_{\theta}=B_{0} J_{1}(\alpha R) /\left[\left(1+t / t_{0}\right)^{2}+K /\left(c_{1}^{2} t_{0}^{2}\right)\right], \\
& B_{z}=B_{0} J_{0}(\alpha R) /\left[\left(1+t / t_{0}\right)^{2}+K /\left(c_{1}^{2} t_{0}^{2}\right)\right],
\end{aligned}
$$

where $B_{0}=B_{1} /\left(c_{1} t_{0}^{2}\right), \alpha=\alpha_{1} /\left(c_{1} t_{0}^{2}\right)^{1 / 2}$, and $R=$ $r /\left[\left(1+t / t_{0}\right)^{2}+K /\left(c_{1}^{2} t_{0}^{2}\right)\right]^{1 / 2}$. We assumed $\gamma=5 / 3$ here to get similar expressions for the magnetic field to the previous solution.

The limit $K \rightarrow 0$ is considered here. This limit corresponds to the low $\beta$ limit. The $\beta$ value of interplanetary flux ropes is usually significantly less than 1 (Burlaga et al., 1981). Then,

$$
\begin{gathered}
B_{\theta}=B_{0} J_{1}\left(\alpha r /\left(1+t / t_{0}\right)\right) /\left(1+t / t_{0}\right)^{2}, \\
B_{z}=B_{0} J_{0}\left(\alpha r /\left(1+t / t_{0}\right)\right) /\left(1+t / t_{0}\right)^{2} .
\end{gathered}
$$

$B_{z}$ is expressed in the same way as in the model excluding elongation. We shall compare the model excluding elongation (Eqs. (44) and (45)) and the new model including elongation (Eqs. (48) and (49)).

We used the key parameter data obtained by the WIND spacecraft. The WIND spacecraft observes the interplanetary magnetic field and plasma parameters in the solar wind (Lepping et al., 1995; Ogilvie et al., 1995). We used the data on magnetic fields, proton density, bulk velocity, and proton thermal velocity of the solar wind from 1 Jan. to 31 Dec. in 1995. We examine the 31 flux ropes selected by Shimazu and Marubashi (2000) using 60-min and 30-min values of these key parameters. To fit the data to models, we used the same method as that used by Marubashi (1997), who employed the Osherovich et al. (1995) self-similar solution. We compared the difference ( $\chi^{2}$ value) between the model magnetic field and the observational one to determine which model is better. The $\chi^{2}$ value is defined by

$$
\begin{aligned}
\chi^{2}=\frac{1}{N} \sum\{ & \left(b_{x}^{O}-b_{x}^{M}\right)^{2} \\
& \left.+\left(b_{y}^{O}-b_{y}^{M}\right)^{2}+\left(b_{z}^{O}-b_{z}^{M}\right)^{2}\right\},
\end{aligned}
$$

where $b_{i}^{O}$ is the $i$-th component of the interplanetary magnetic fields, $b_{i}^{M}$ is the $i$-th component of the model magnetic fields obtained after fitting, and $N$ is the number of data points (Lepping et al., 1990). Both $b_{i}^{O}$ and $b_{i}^{M}$ are unit normalized. Note that this $\chi^{2}$ is different from the $\chi$ defined in Subsection 2.1.

After fitted the magnetic field to the model, we fitted the velocity. To represent the elongation, we must introduce new geometric parameters. In this paper we introduce two geometric parameters $z_{1}$ and $z_{2}$. The new parameter $z_{1}$ is the axial distance from the center of the flux rope to the location of the spacecraft encounter and $z_{2}$ is the one from the center to the location where the spacecraft detached from the flux rope (Fig. 3). These parameters are determined as a result of the fitting to minimize the velocity difference between the model and the observation.

\subsection{Results}

The magnetic field data showed that $B_{\theta}=B_{0} J_{1}(\alpha r /(1+$ $\left.\left.t / t_{0}\right)\right) /\left(1+t / t_{0}\right)$ type (excluding elongation) was better in 17 events, and that $B_{\theta}=B_{0} J_{1}\left(\alpha r /\left(1+t / t_{0}\right)\right) /\left(1+t / t_{0}\right)^{2}$ type (including elongation) was better in 14 events. Thus, approximately half of the flux ropes were fitted better to the model including elongation and the remainders were fitted better to the model excluding elongation.

Figure 4 shows an event on Feb. 8, 4:30-19:30. When we use the model excluding elongation, the fitting parameters for this event are: $U_{s w}=414.3 \mathrm{~km} / \mathrm{s}$ (bulk velocity), $B_{0}=$ $-22.2 \mathrm{nT}, R_{0}=0.0922 \mathrm{AU}$ (radius of the flux rope at spacecraft encounter), $\theta_{1}=-10.8$ degrees (ecliptic latitude angle of the symmetry axis), $\phi_{1}=64.6$ degrees (ecliptic 

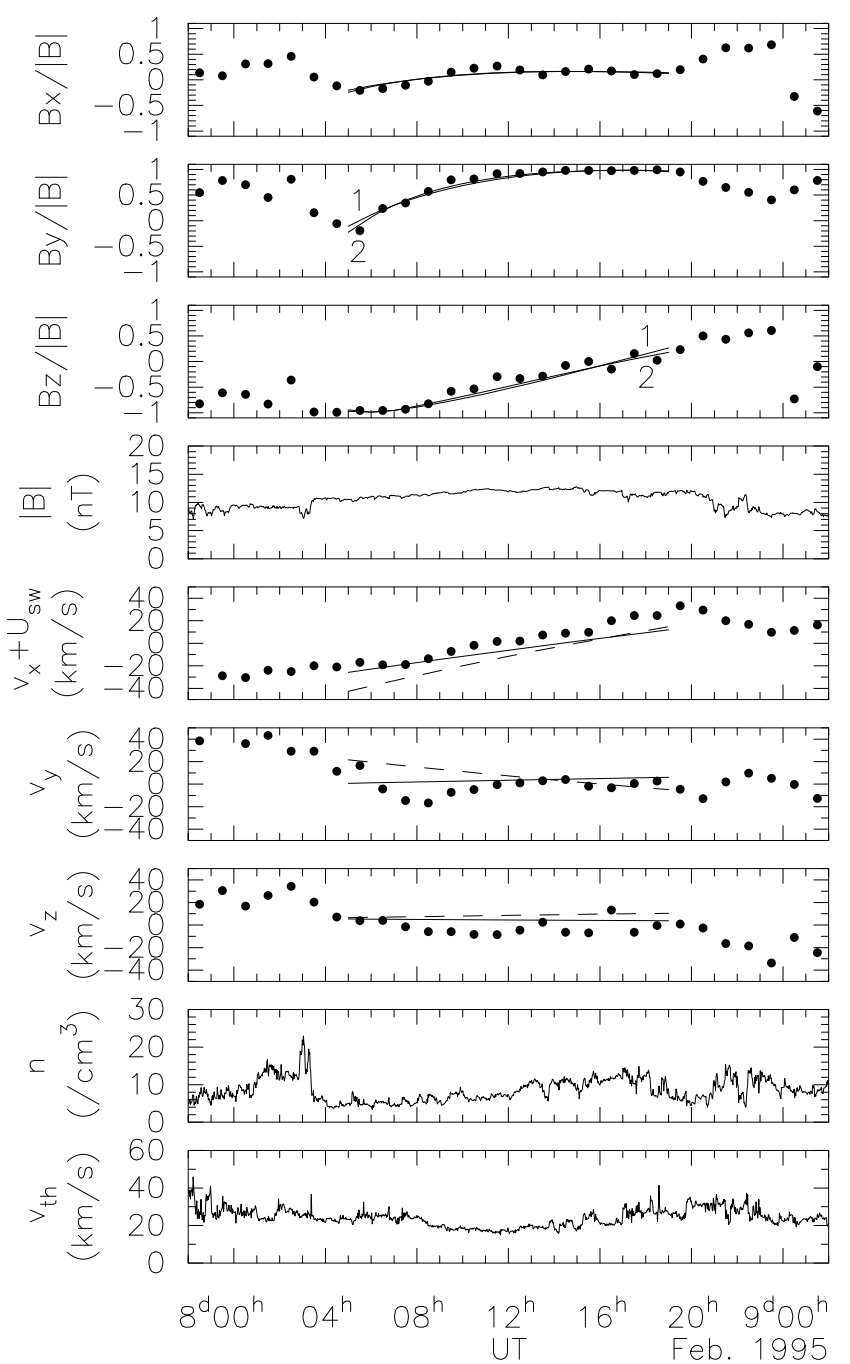

Fig. 4. Flux rope observed by the WIND spacecraft on Feb. 8, 1995. $n$ is the proton number density, and $v_{t h}$ is the thermal velocity of protons. The GSE coordinates are used. The dots are the 60-min averages of the observation, the lines numbered 1 or dashed lines are the fitted model fields excluding elongation, and the lines numbered 2 or solid lines are the fitted model fields including elongation.

longitude angle of the symmetry axis measured from the line connecting the sun and the earth), $p=0.224$ (impact parameter), and $\chi^{2}=0.0290$.

When we use the model including elongation, the fitting parameters for this event are: $U_{s w}=414.3 \mathrm{~km} / \mathrm{s}, B_{0}=$ $-22.6 \mathrm{nT}, R_{0}=0.0802 \mathrm{AU}, \theta_{1}=-18.0$ degrees, $\phi_{1}=65.5$ degrees, $p=0.264$, and $\chi^{2}=0.0234$. In this case fitting is better when the effect of elongation is included.

In the panels of the magnetic fields in Fig. 4, the lines numbered 1 are the fitted model fields excluding elongation, and the lines numbered 2 are the fitted model fields including elongation. The difference between the two models is slight. The other events also showed that the difference is very small.

However the panels of velocities show that the fitting is improved when the elongation is included. In this case, the value of the new parameters are $z_{1}=-0.030 \mathrm{AU}$ and $z_{2}=-0.026 \mathrm{AU}$.

Figure 5 shows another flux rope event of Oct. 19, 1995,

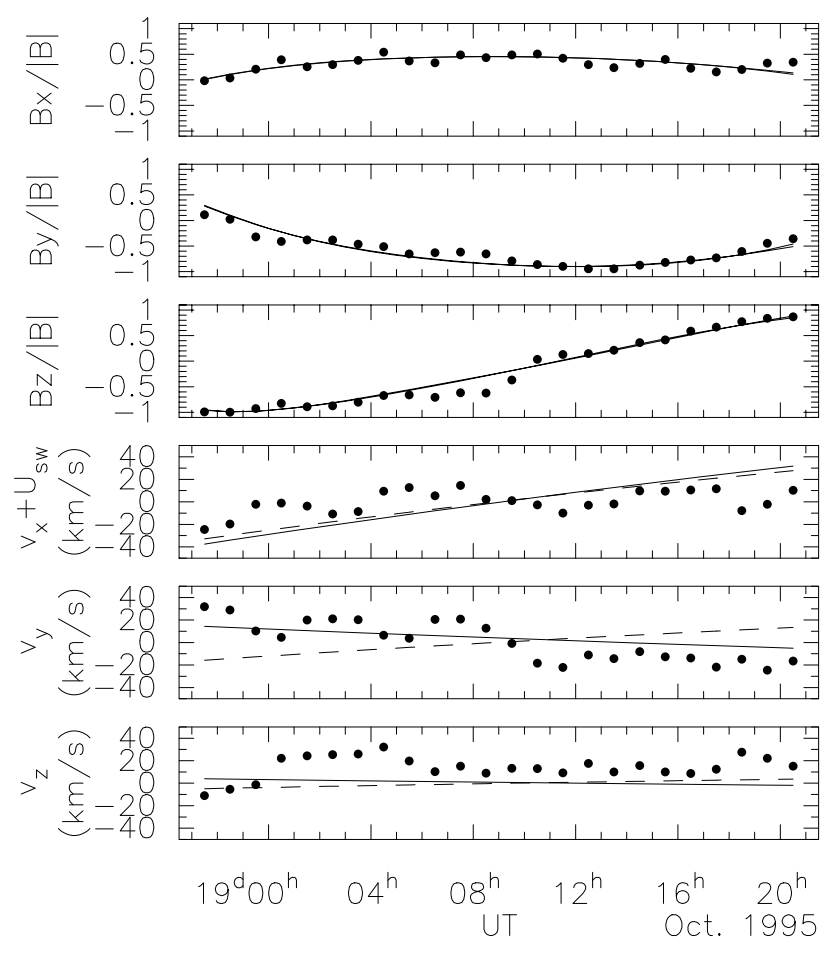

Fig. 5. Flux rope observed by the WIND spacecraft on Oct. 19, 1995. The GSE coordinates are used. The dots are the 60-min averages of the observation, the dashed lines are the fitted model fields excluding elongation, and the solid lines are the fitted model fields including elongation.

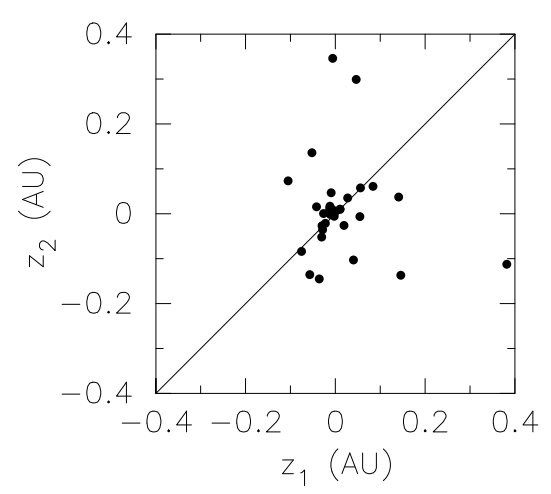

Fig. 6. Distribution of $z_{1}$ and $z_{2}$ for the 31 flux ropes. $\left|z_{2}-z_{1}\right|$ is the vertical distance from each dot to the $z_{2}=z_{1}$ line.

which was analyzed by Larson et al. (1997). This figure also shows that the difference in the magnetic field between the two models is slight. However the velocity fitting is better, especially in the $y$ component, when the elongation and the new parameters are considered. In this case $z_{1}=-0.11 \mathrm{AU}$ and $z_{2}=0.073 \mathrm{AU}$. Since the variation in the velocity is not as smooth as that in the magnetic field, the fitting of the velocity is worse than that of the magnetic field.

We plotted the values of $z_{1}$ and $z_{2}$ for the 31 flux ropes to examine the fitting validity (Fig. 6). $\left|z_{2}-z_{1}\right|$, which is the vertical distance from each dot to the $z_{2}=z_{1}$ line in Fig. 6, is the axial component of the spacecraft trajectory in the flux rope (see Fig. 3). This value obtained from the fitting is almost the same scale as the radius of flux ropes, which is consistent with the assumption of the isotropic expansion. 


\subsection{Discussion}

The magnetic field data showed that approximately half of the flux ropes were fitted better to the model including elongation and that the remainders were fitted better to the model excluding elongation when we examined 31 flux ropes obtained from the WIND key parameters for the year 1995. In fact, the difference between these two models was slight. When we observe near the sun, where the expansion is rapid, a large difference may arise. Using data observed near the sun is a task for the future.

We omitted the term including $K$ in Eqs. (46) and (47) in order to compare the two models. However, this term may have to be considered because fitting results often show unrealistic $t_{0}$ values, which represents the propagation time of a flux rope from the sun to an observation point. There is the possibility of getting realistic $t_{0}$ values by considering this term.

Our model did not include the following effects that should be considered in real magnetic clouds: 1) Magnetic clouds are not two-dimensional straight cylindrical structures. At least some magnetic clouds have their foot points connected to the sun (e.g., Larson et al., 1997). In our model, the curvature of the axis was not included. 2) Cargill et al. (1996)'s computer simulation of a magnetic cloud showed that an initial circle cross section is transformed into a noncircular cross section during propagation. The noncircular cross section was not considered in our model.

\section{Summary}

We showed a theoretical basis for the fact that $\gamma$ can be larger than 1 when a cylindrical flux rope expands selfsimilarly by considering the effects of axial elongation as well as radial expansion. We found a new class of solutions for two-dimensional MHD equations that satisfies the condition that a cylindrical flux rope can expand self-similarly for any $\gamma$ value. This new class of solutions puts new light to the very discussed problem on the relation between $\gamma$ and the expansion. Mainly it emphasizes a role of flux rope elongation for its expansion. This new solution modified the azimuthal component of the magnetic field from Eq. (44) to Eq. (48). This solution also showed that the flux rope expands maintaining a force-free state.

The models including elongation and excluding elongation were compared with observations. The difference in the fitting results of the magnetic field between these two models was slight. However the fitting of the velocity was improved when elongation was included and when new geometric parameters $z_{1}$ and $z_{2}$, which were necessary to represent the elongation, were introduced. The values of these parameters were almost the same scale as the radius of flux ropes, which was consistent with the assumption of the isotropic expansion.

As we close, we would like to emphasize one important aspect of this work. The new solution represents an exact time-dependent two-dimensional solution of ideal MHD equations and therefore it is suitable for tests of current or developing MHD numerical codes. There are not many such solutions in literature. The former solution was only onedimensional, which was used to test a code by Vandas and Odstrčil (2000).
Acknowledgments. The WIND key parameter data on the magnetic field were provided by Dr. Ronald P. Lepping (NASA Goddard Space Flight Center) and the WIND MFI team. The WIND key parameter data on the proton density, bulk velocity, and proton thermal velocity were provided by Dr. Keith W. Ogilvie (NASA Goddard Space Flight Center) and the WIND SWE team. The authors thank Dr. Katsuhide Marubashi (Communications Research Laboratory, Japan), and Dr. Shin-ichi Watari (Communications Research Laboratory, Japan) for their stimulating and insightful comments and suggestions during the course of this work. H. S. is supported by the JSPS overseas research fellowship. M. V. was supported by project $\mathrm{S} 1003006$ from AV ČR.

\section{Note}

We were notified that a paper which deals with a similar topic would be submitted to Phys. Rev. Lett., by Berdichevsky et al. (2002). It should be noted here that our manuscript was completed and submitted independently of their manuscript.

\section{References}

Berdichevsky, D. B., R. P. Lepping, and C. J. Farrugia, On geometric considerations of the evolution of magnetic flux reopes, Phys. Rev. Lett., 2002 (submitted).

Bernstein, I. B. and R. M. Kulsrud, On the explosion of a supernova into the interstellar magnetic field. I, Astrophys. J., 142, 479-490, 1965.

Burlaga, L. F., Magnetic clouds and force-free fields with constant alpha, $J$. Geophys. Res., 93, 7217-7224, 1988.

Burlaga, L., E. Sittler, F. Mariani, and R. Schwenn, Magnetic loop behind an interplanetary shock: Voyager, Helios, and IMP 8 observations, $J$. Geophys. Res., 86, 6673-6684, 1981.

Cargill, P. J., J. Chen, D. S. Spicer, and S. T. Zalesak, Magnetohydrodynamic simulations of the motion of magnetic flux tubes through a magnetized plasma, J. Geophys. Res., 101, 4855-4870, 1996.

Chen, J., Theory of prominence eruption and propagation: Interplanetary consequences, J. Geophys. Res., 101, 27,499-27,519, 1996.

Chen, J. and D. A. Garren, Interplanetary magnetic clouds: Topology and driving mechanism, Geophys. Res. Lett., 20, 2319-2322, 1993.

Farrugia, C. J., L. F. Burlaga, V. A. Osherovich, and R. P. Lepping, A comparative study of dynamically expanding force-free, constant-alpha magnetic configurations with applications to magnetic clouds, in Solar Wind Seven, edited by E. Marsch and R. Schwenn, COSPAR, vol. 3, pp. 611-614, Pergamon, New York, 1992.

Farrugia, C. J., L. F. Burlaga, V. A. Osherovich, I. G. Richardson, M. P. Freeman, R. P. Lepping, and A. J. Lazarus, A study of an expanding interplanetary magnetic cloud and its interaction with the earth's magnetosphere: The interplanetary aspect, J. Geophys. Res., 98, 7621-7632, 1993.

Gibson, S. E. and B. C. Low, A time-dependent three-dimensional magnetohydrodynamic model of the coronal mass ejection, Astrophys. J., 493, 460-473, 1998.

Goldstein, H., On the field configuration in magnetic clouds, Solar Wind Five, NASA Conf. Publ., 2280, 731-733, 1983.

Gosling, J. T., On the determination of electron polytrope indices within coronal mass ejections in the solar wind, J. Geophys. Res., 104, 19,85119,857, 1999.

Gosling, J. T., P. Riley, and R. M. Skoug, Reply, J. Geophys. Res., 106, 3709-3713, 2001.

Kulsrud, R. M., I. B. Bernstein, M. Kruskal, J. Fanucci, and N. Ness, On the explosion of a supernova into the interstellar magnetic field. II, Astrophys. J., 142, 491-506, 1965.

Kumar, A., and D. M. Rust, Interplanetary magnetic clouds, helicity conservation, and current-core flux-ropes, J. Geophys. Res., 101, 15,66715,684, 1996.

Larson, D. E., R. P. Lin, J. M. McTiernan, J. P. McFadden, R. E. Ergun, M. McCarthy, H. Rème, T. R. Sanderson, M. Kaiser, R. P. Lepping, and J. Mazur, Tracing the topology of the October 18-20, 1995, magnetic cloud with $\sim 0.1-10^{2} \mathrm{keV}$ electrons, Geophys. Res. Lett., 24, 1911-1914, 1997.

Lepping, R. P., J. A. Jones, and L. F. Burlaga, Magnetic field structure of interplanetary magnetic clouds at $1 \mathrm{AU}, J$. Geophys. Res., 95, 11,95711,965, 1990.

Lepping, R. P., M. H. Acũna, L. F. Burlaga, W. M. Farrell, J. A. Slavin, K H. Schatten, F. Mariani, N. F. Ness, F. M. Neubauer, Y. C. Whang, J. B. Byrnes, R. S. Kennon, P. V. Panetta, J. Scheifele, and E. M. Worley, The WIND magnetic field investigation, Space Sci. Rev., 71, 207-229, 1995. 
Low, B. C., Self-similar magnetohydrodynamics. I. The $\gamma=4 / 3$ polytrope and the coronal transient, Astrophys. J., 254, 796-805, 1982.

Marubashi, K., Structure of the interplanetary magnetic clouds and their solar origins, Adv. Space Res., 6(6), 335-338, 1986.

Marubashi, K., Interplanetary magnetic flux ropes and solar filaments, in Coronal Mass Ejections (Geophysical monograph 99), edited by $\mathrm{N}$ Crooker, J. A. Joselyn, and J. Feynman, pp. 147-156, American Geophysical Union, Washington, D.C., 1997.

Odstrčil, D. and V. J. Pizzo, Three-dimensional propagation of coronal mass ejections (CMEs) in a structured solar wind flow 1. CME launched within the streamer belt, J. Geophys. Res., 104, 483-492, 1999.

Ogilvie, K. W., D. J. Chornay, R. J. Fritzenreiter, F. Hunsaker, J. Keller, J. Lobell, G. Miller, J. D. Scudder, E. C. Sittler, Jr., R. B. Torbert, D. Bodet, G. Needell, A. J. Lazarus, J. T. Steinberg, J. H. Tappan, A. Mavretic, and E. Gergin, SWE, a comprehensive plasma instrument for the WIND spacecraft, Space Sci. Rev., 71, 55-77, 1995.

Osherovich, V., Comment on "On the determination of electron polytrope indices within coronal mass ejections in the solar wind", J. Geophys. Res., 106, 3703-3707, 2001.

Osherovich, V. A., C. J. Farrugia, and L. F. Burlaga, Nonlinear evolution of magnetic flux ropes 1. Low-beta limit, J. Geophys. Res., 98, 13,22513,231, 1993a.

Osherovich, V. A., C. J. Farrugia, L. F. Burlaga, R. P. Lepping, J. Fainberg, and R. G. Stone, Polytropic relationship in interplanetary magnetic clouds, J. Geophys. Res., 98, 15,331-15,342, 1993b.

Osherovich, V. A., C. J. Farrugia, and L. F. Burlaga, Nonlinear evolution of magnetic flux ropes 2. Finite beta plasma, J. Geophys. Res., 100, 12,307$12,318,1995$.

Shimazu, H. and K. Marubashi, New method for detecting interplanetary flux ropes, J. Geophys. Res., 105, 2365-2373, 2000

Skoug, R. M., W. C. Feldman, J. T. Gosling, D. J. McComas, and C. W. Smith, Solar wind electron characteristics inside and outside coronal mass ejections, J. Geophys. Res., 105, 23,069-23,084, 2000a.

Skoug, R. M., W. C. Feldman, J. T. Gosling, D. J. McComas, D. B. Reisenfeld, C. W. Smith, R. P. Lepping, and A. Balogh, Radial variation of solar wind electrons inside a magnetic cloud observed at 1 and 5 AU, J. Geophys. Res., 105, 27,269-27,275, 2000 b.

Tsurutani, B. T., W. D. Gonzalez, F. Tang, S. I. Akasofu, and E. J. Smith, Origin of interplanetary southward magnetic fields responsible for major magnetic storms near solar maximum (1978-1979), J. Geophys. Res., 93, 8519-8531, 1988.

Vandas, M. and D. Odstrčil, Magnetic cloud evolution: A comparison of analytical and numerical solutions, J. Geophys. Res., 105, 12,605-12,616, 2000 .

Vandas, M., S. Fischer, M. Dryer, Z. Smith, and T. Detman, Simulation of magnetic cloud propagation in the inner heliosphere in two-dimensions 1 . A loop perpendicular to the ecliptic plane, J. Geophys. Res., 100, 12,28512,292, 1995.

Vandas, M., S. Fischer, M. Dryer, Z. Smith, and T. Detman, Parametric study of loop-like magnetic cloud propagation, J. Geophys. Res., 101, 15,645-15,652, 1996.

Wu, S. T., W. P. Guo, and M. Dryer, Dynamical evolution of a coronal streamer-flux rope system II. A self-consistent non-planar magnetohydrodynamic simulation, Solar Phys., 170, 265-282, 1997.

H. Shimazu (e-mail: shimazu@geophys.washington.edu) and M. Vandas (e-mail: vandas@ig.cas.cz) 